\title{
Hippocampus-Specific fMRI Group Activation Analysis with Continuous M-Reps
}

\author{
Paul A. Yushkevich ${ }^{1}$, John A. Detre ${ }^{2}$, Kathy Z. Tang ${ }^{2}$, Angela Hoang ${ }^{2}$, \\ Dawn Mechanic-Hamilton ${ }^{2}$, María A. Fernández-Seara ${ }^{2}$, Marc Korczykowski ${ }^{2}$, \\ Hui Zhang ${ }^{1}$, and James C. Gee ${ }^{1}$ \\ ${ }^{1}$ Penn Image Computing and Science Laboratory, University of Pennsylvania \\ ${ }^{2}$ Center for Functional Neuroimaging, University of Pennsylvania
}

\begin{abstract}
A new approach to group activation analysis in fMRI studies that test hypotheses focused on specific brain structures is presented and used to analyze hippocampal activation in a visual scene encoding study. The approach leverages the $c m$-rep method [10] to normalize hippocampal anatomy and project intra-subject hippocampal activation maps into a common reference space, eliminating normalization errors inherent in whole-brain approaches and guaranteeing that peaks detected in the random effects activation map are indeed associated with the hippocampus. When applied to real fMRI data, the method detects more significant hippocampal activation than the established whole-brain method.
\end{abstract}

\section{Introduction}

Functional MRI has revolutionized many fields of science, with numerous studies published each year. Although fMRI studies address a variety of hypotheses and use a variety of experimental designs, most employ a fairly rigid image processing and statistical analysis pipeline, defined by the Statistical Parametric Mapping (SPM) paradigm [4. In SPM, group activation analysis involves normalizing each subject's brain anatomy to a whole-brain template, performing random effects (RFX) analysis in the space of this template, and assigning anatomical labels to supra-threshold clusters and peaks in the resulting statistical map.

This paper proposes an alternative analysis pipeline aimed at studies that test hypotheses focused on specific brain structures. In particular, we focus on analyzing activation in the hippocampus, a frequently studied structure responsible for episodic memory encoding. Using the $\mathrm{cm}$-rep method [10, we fit a common shape-based coordinate system to the hippocampus in a manner that is consistent across subjects. This system allows us to project each subject's hippocampal activation values into a common reference frame. By performing RFX analysis in this frame, we eliminate normalization errors stemming from whole-brain registration and ensure that clusters and peaks in the RFX map can be labeled as hippocampal activation with high degree of certainty, as opposed to SPM clusters and peaks, whose localization accuracy depends on the quality of the normalization to an anatomical atlas. 
Table 1. Protocol implemented on the 3 Tesla Siemens Trio for the fMRI study

\begin{tabular}{llrrr} 
SCAN & Resolution $\left(\mathrm{mm}^{3}\right)$ & TR $(\mathrm{ms})$ & TE $(\mathrm{ms})$ & FA $\left(^{\circ}\right)$ \\
\hline$T_{1}$ MP-RAGE & $0.977 \times 0.977 \times 1$ & 1620 & 3.87 & 15 \\
EPI I & $3.0 \times 3.0 \times 3.0$ & 3000 & 30 & 90 \\
EPI II & $2.5 \times 2.5 \times 2.5$ & 3000 & 30 & 90
\end{tabular}

Other alternatives to SPM have also been proposed, including, but not limited to, methods that study activation on the cortical surface [3, 8, and the hippocampus unrolling approach by Zeineh et al. [11], which is related to our approach, but differs from it significantly, since it uses MR images with highly anisotropic voxels and produces $2 \mathrm{D}$ hippocampal activation maps.

\section{Materials and Methods}

\subsection{Subjects, Experimental Design and Data Acquisition}

In a recent block design fMRI study conducted by the Center for Functional Neuroimaging, structural and functional brain scans were acquired from 30 healthy young adults at 3 Tesla. Table 1 summarizes the protocol, which includes a highresolution T1 scan and two EPI scans with isotropic voxels $2.5 \mathrm{~mm}$ and $3 \mathrm{~mm}$ wide, acquired in randomized order in order to study the tradeoff between signalto-noise ratio and partial volume effects. During each EPI scan, subjects were presented with novel complex visual scenes (photographs of people engaged in various activities, industrial landscape, etc.) and asked to remember them for a subsequent test. Scenes were presented in blocks of ten, with $3600 \mathrm{~ms}$ per stimulus and $400 \mathrm{~ms}$ inter-stimulus interval. To provide a baseline for analysis, subjects were shown scrambled scenes after each block, and asked to examine them as they would a normal picture. In the testing phase, subjects were shown another set of scenes, some previously seen and others novel, and asked to classify them as such; all subjects responded correctly more than $80 \%$ of the time.

\subsection{Intra-subject Activation Analysis}

SPM2 and VoxBo software were used to generate intra-subject statistical maps. fMRI sequences were motion-corrected and co-registered with T1 data (analysis was also repeated without such co-registration). fMRI data were spatially smoothed with $9 \mathrm{~mm}$ FWHM Gaussian kernels. Time series were smoothed temporally to remove frequencies above the task frequency and convolved with a canonical model of the hemodynamic response function. Box car task reference functions were also convolved with the hemodynamic response function and used as covariates in the general linear model, along with a global signal covariate, to produce statistical parametric maps. For each subject, maps of $z$-statistics corresponding to voxel-wise one-tail $t$-tests were computed. 


\subsection{Random Effects Analysis with Whole-Brain Normalization}

RFX analysis is used to make inferences about a population on the basis of fMRI data from a group of subjects [6]. RFX statistical maps are computed by (1) normalizing each subject's brain to a template, (2) using the normalization parameters to warp intra-subject activation maps into the space of the template, and (3) performing a second-level $t$-test at each voxel in this space. Normalization was implemented, as in many studies, by warping each T1 image to the Montreal Neurological Institute template [2] using SPM2, which first estimates a 12-parameter affine transformation, followed by a non-linear registration based on the discrete cosine basis 11. Limitations of this approach include suboptimal normalization of the hippocampus due to its relatively low contribution to the registration objective function, which is integrated over the whole brain, as well as the uncertainty associated with assigning anatomical labels to peaks in the RFX statistical map. The $c m$-rep approach aims to overcome these limitations.

\subsection{Hippocampus-Centric Random Effects Analysis with CM-Reps}

The $c m$-reps approach is the continuous analog of the m-rep method by Pizer et al. 7. Anatomical structures are represented by fitting a deformable model to binary segmentations of the structure (theoretically, fitting directly to anatomical images is also possible, but not implemented in this paper). The deformable model has a special property: it describes a geometric object in terms of the relationship between its skeleton and its boundary, and it preserves the branching topology of the skeleton during deformation. Specifically, a $\mathrm{cm}$-rep model defines the skeleton as a single manifold with boundary $\mathbf{m}$ along which a certain scalar field $\rho$ is defined. Both $\mathbf{m}$ and $\rho$ are defined parametrically as

$$
\mathbf{m}(\mathbf{u})=\sum_{i=0}^{N} \mathbf{m}_{i} f_{i}(\mathbf{u}) ; \quad \rho(\mathbf{u})=\sum_{i=0}^{M} \rho_{i} f_{i}(\mathbf{u}),
$$

where $\left\{f_{i}\right\}$ is some basis (e.g., b-splines, Fourier harmonics) and $\mathbf{m}_{i} \in \mathbb{R}^{3}$ and $\rho_{i} \in \mathbb{R}$ are basis function coefficients that can be used to deform $\mathbf{m}$ and change $\rho$. The parameter vector $\mathbf{u}=\left(u^{1}, u^{2}\right)$ is defined on some regular domain $\Omega \in \mathbb{R}^{2}$. From $\mathbf{m}$ and $\rho$, a radius function $R$ is derived by solving the following partial differential equation:

$$
\triangle_{\mathbf{m}} R^{2}=\rho \quad \text { subj. to } \quad R\left(1-\left\|\nabla_{\mathbf{m}} R\right\|^{2}\right)=0 \quad \text { on } \partial \Omega,
$$

where $\triangle_{\mathbf{m}}$ and $\nabla_{\mathbf{m}}$ denote the Laplace-Beltrami and gradient operators on the manifold $\mathbf{m}$. The boundary condition in (11) corresponds to the equality constraint that is satisfied by single-manifold skeletons of generic objects [9]. The PDE is well-behaved in practice, and solutions, as well as their gradients, can be found efficiently using the Finite Differences Method.

Given the manifold $\mathbf{m}$ and having solved for the field $R$, we can apply inverse skeletonization to derive a parametric expression for the boundary of the object 

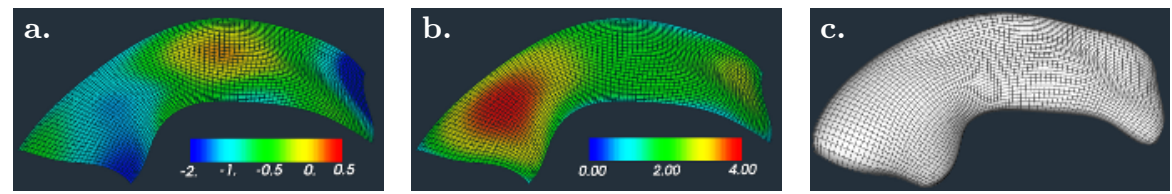

Fig. 1. Three steps of constructing a $c m-r e p$. a. Medial manifold $\mathbf{m}(\mathbf{u})$ with a color plot of the scalar field $\rho(\mathbf{u})$. b. The radius scalar field $R(\mathbf{u})$ computed by solving the Poisson PDE (1) on the medial manifold. c. Boundary surface $\mathbf{b}^{ \pm}(\mathbf{u})$ resulting from inverse skeletonization.

$\mathcal{O}$ whose skeleton is $\{\mathbf{m}, R\}$ (the skeleton is the set of centers and radii of all maximal inscribed balls in the object). In work that has not yet been published, we give a list of sufficient conditions for which we prove that the inverse skeletonization problem is well-posed; these include the boundary condition in (1) and some inequality constraints. When these constraints hold, the solution to inverse skeletonization is given by the following formula:

$$
\mathbf{b}^{ \pm}=\mathbf{m}+R \mathbf{U}^{ \pm}, \quad \text { where } \quad \mathbf{U}^{ \pm}=-\nabla_{\mathbf{m}} R \pm \sqrt{1-\left\|\nabla_{\mathbf{m}} R\right\|^{2}} \mathbf{N}_{\mathbf{m}},
$$

where $\mathbf{N}_{\mathbf{m}}$ is the unit normal to $\mathbf{m}$. Manifolds $\mathbf{b}^{+}$and $\mathbf{b}^{-}$given by the above expression are two surface patches that lie on the opposite sides of $\mathbf{m}$ and together form the boundary of the object $\mathcal{O}$. For every $\mathbf{u} \in \Omega$, the ball with center $\mathbf{m}(\mathbf{u})$ and radius $R(\mathbf{u})$ is a maximal inscribed ball in $\mathcal{O}$ and is tangent to $\partial \mathcal{O}$ at the points $\mathbf{b}^{-}(\mathbf{u})$ and $\mathbf{b}^{+}(\mathbf{u})$. Vectors $\mathbf{U}^{ \pm}$project from the center of the ball towards these points of tangency and have unit length; hence they form the unit normal vector field to $\partial \mathcal{O}$. It is easy to verify that the boundary condition in (1) ensures that $\mathbf{U}^{+}$and $\mathbf{U}^{-}$coincide for $\mathbf{u} \in \partial \Omega$ and that patches $\mathbf{b}^{+}$and $\mathbf{b}^{-}$ together form a closed surface. An example $c m$-rep model is shown in Fig. 1 . first as the inputs $\mathbf{m}$ and $\rho$, next as the field $R$ obtained by solving (1), and last sa the boundary surface computed by inverse skeletonization.

We refer the reader to [9, 10] for the details of the deformable modeling approach used to fit $c m$-rep models to actual structures. It follows the Bayesian framework, with an image-driven likelihood term and prior terms that enforce inequality constraints that permit inverse skeletonization, impose geometric correspondence by minimizing distortion in the area element of $\mathbf{m}$, and incorporate a shape prior. Since the deformable model is restricted to have a single-manifold skeleton, it matches real-world objects with some inherent level of error. In [9] we report high accuracy when fitting $\mathrm{cm}$-rep models to hippocampi from an 82 -subject schizophrenia study. In more recent unpublished results, the average overlap is $95.0 \%$ and the average boundary displacement error is $0.168 \mathrm{~mm}$.

A key feature of cm-reps discussed in [10] is the ability to impose a shapebased coordinate system on the interiors of structures. Every point $\mathbf{x}$ inside the cm-rep boundary can be assigned a triple of values $u^{1}, u^{2}, \xi$ that satisfy

$$
\mathbf{x}(\mathbf{u}, \xi)=\mathbf{m}(\mathbf{u})+|\xi| R(\mathbf{u}) \mathbf{U}^{\operatorname{sgn}(\xi)}(\mathbf{u})
$$



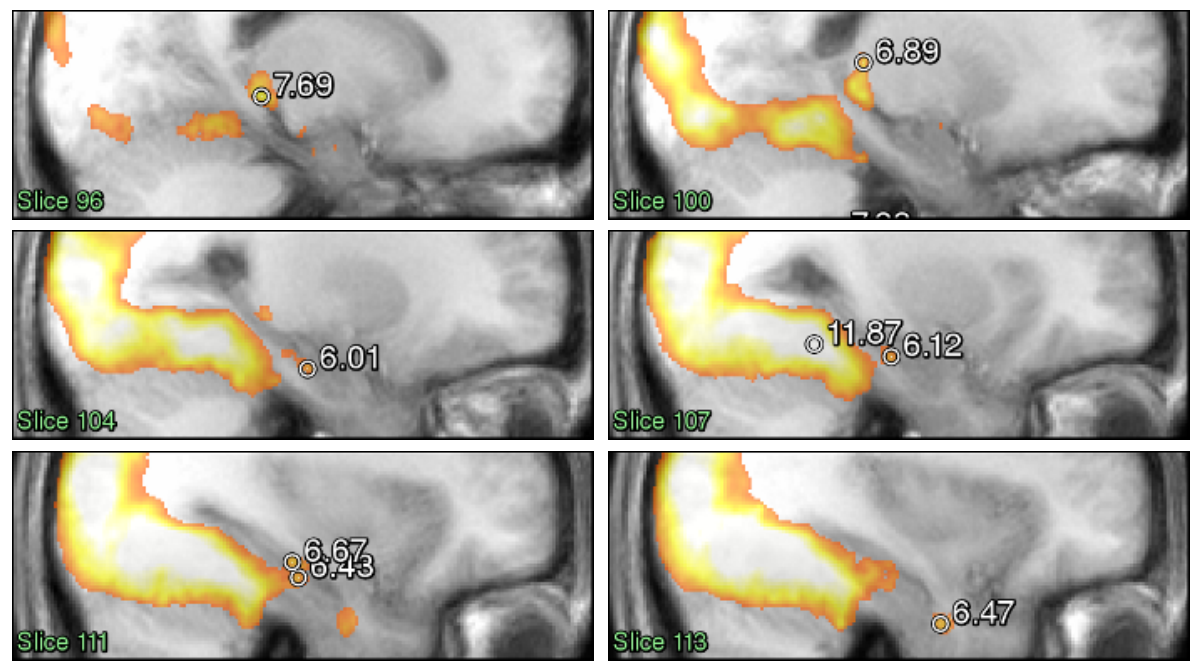

Fig. 2. Selected sagittal slices through the RFX activation map computed using wholebrain normalization, superimposed over the average anatomical image. Please see also the corresponding color Quicktime movie: whole_brain_rfx.mov.

The assignment is unique, except in the case $\mathbf{u} \in \partial \Omega$, when $\mathbf{x}(\mathbf{u}, \xi)=\mathbf{x}(\mathbf{u},-\xi)$. This assignment can be used to map the interior of the object into a reference space $\Omega \times[-1,1]$. This mapping has an important feature: the distance from $\mathbf{x}(\mathbf{u}, \xi)$ to the $c m$-rep boundary is given by $(1-|\xi|) R(\mathbf{u})$, so line segments normal to the $c m-r e p$ boundary are mapped to vertical line segments in the reference space. Points on the $c m$-rep skeleton are mapped to the plane $\xi=0$ in the reference space and points on the boundary are mapped to planes $\xi= \pm 1$.

This coordinate system was used to perform hippocampus-specific RFX analysis of activation data. The right hippocampus was outlined manually in T1 images of 18 subjects. A $\mathrm{cm}$-rep template was fitted to the resulting binary segmentations. Intra-subject activation $z$-maps were sampled in the $c m$-rep shapebased coordinate system, i.e., transformed into the $u^{1}, u^{2}, \xi$ space. RFX analysis was performed by computing a one-tail $t$-test on the $z$-statistics at each point in the coordinate system. Thus, the analysis was limited just to the hippocampus region. For visualization purposes, the $t$-statistic field resulting from this RFX analysis was projected back into the image space of one of the subjects.

\section{Results}

Fig. 2 shows the RFX $t$-map obtained using whole-brain analysis. The map is superimposed over a 'mean anatomy' image, computed by averaging the subjects' T1 images following SPM2 normalization. The fuzziness of this image stems 

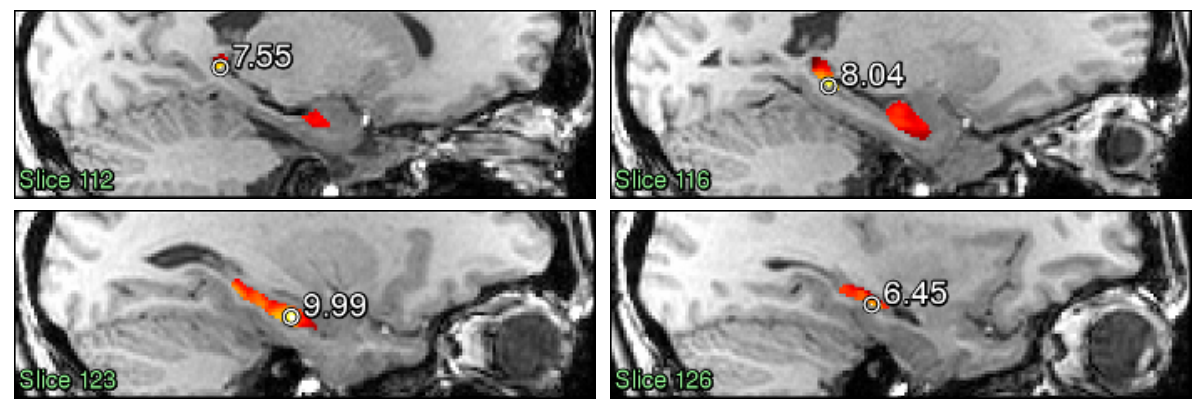

Fig. 3. Selected sagittal slices through the hippocampal RFX activation map computed in the $c m$-rep coordinate system, projected back into the anatomical space of one subject and superimposed over this subject's anatomical image. Please also see the corresponding color Quicktime movie: cmrep_rfx.mov.

from whole-brain normalization error, which is significant: the average pairwise overlap between subjects' hippocampus segmentations warped into the space of the template is $53 \%$. The RFX map in Fig. 2 is threshholded at $t=5$, and peaks near the right hippocampus are highlighted. The locations and labels of the peaks, looked up in the Talairach atlas, are listed in Table 2, Such labeling carries a degree of uncertainty because of normalization error and low resolution of the atlas. For each peak in Table 2, we indicate the probability that it lies in the hippocampus, based on the number of hippocampus masks (warped into the template space) overlapping its location. The fact that these probabilities do not always agree with the anatomical labels underscores the uncertainty associated with anatomical localization of whole-brain RFX peaks.

To address possible concerns about the use of rigid registration for EPI/T1 alignment, the above experiment was repeated without registration. In $3 \mathrm{~mm}^{3}$ fMRI data, the maximal $t$-value observed in the RFX map increased from 16.08 to 16.62 with registration, and the size of the supra-threshold region $t \geq 6.0$ increased from $102 \mathrm{~cm}^{3}$ to $112 \mathrm{~cm}^{3}$. The results for $2.5 \mathrm{~mm}^{3}$ data are analogous.

The results of $c m$-rep-based RFX analysis for $3 \mathrm{~mm}^{3} \mathrm{fMRI}$ are shown in Fig. 3 . The underlying anatomical image is a T1 scan of one of the subjects. The RFX $t$-map, computed in the $c m$-rep reference space, was projected back into the subject's anatomical space for visualization. The peaks of the $t$-map shown here have larger $t$-values than the peaks in and around the hippocampus shown in Fig. 2. The locations of these peaks within the hippocampus are given in Table 2, Notably, the maximum $t$-values observed in the hippocampus with $\mathrm{cm}$-rep analysis exceed those detected around the hippocampus with whole-brain analysis. The latter, however, may or may not indicate hippocampal activation, due to the errors associated with atlas-based anatomical labeling. The picture is similar for $2.5 \mathrm{~mm}^{3}$ data: $\mathrm{cm}$-rep normalization yields larger hippocampal activation values than SPM, with the former generating peaks $t=7.83,7.43,7.18,6.22$ in the right hippocampus and the latter reporting peak $t=6.90$ in the right hippocampus and $t=7.48,7.47,7.37$ in the right parahippocampal gyrus. 
Table 2. Peak locations in RFX $t$-map computed for $3 \mathrm{~mm}^{3}$ fMRI data using wholebrain and $c m$-rep based normalization. $\mathrm{RC}=$ right cerebrum; $\mathrm{GM}=$ gray matter; $\mathrm{WM}$ $=$ white matter; PHG = parahippocampal gyrus. The column $p(\mathrm{H})$ gives the fraction of hippocampus masks warped to the space of the template which overlap the given peak location.

Peaks in the Whole-Brain RFX $t$-Map

\begin{tabular}{llcr}
\hline Tal. Coord. & Talairach Labels & $t$-Stat & $p(\mathrm{H})$ \\
\hline $16,-31,-2$ & RC, limbic lobe, sub-gyral, *, * & 7.69 & 0 \\
$20,-29,8$ & RC, sub-lobar, thalamus, GM, pulvinar & 6.89 & 0 \\
$31,-22,-9$ & RC, temporal lobe, sub-gyral, GM, hippocampus & 6.67 & 0.94 \\
$33,-6,27$ & RC, limbic lobe, uncus, WM, * & 6.47 & 0 \\
$31,-20,-13$ & RC, limbic lobe, PHG, GM, hippocampus & 6.43 & 0.82 \\
$27,-21,-13$ & RC, limbic lobe, PHG, GM, Brodmann area 6 & 6.12 & 0.94 \\
$24,-17,-17$ & RC, limbic lobe, PHG, WM, * & 6.01 & 0.94
\end{tabular}

Peaks in the Hippocampus-Specific CM-Rep RFX Map

\begin{tabular}{lclc}
\hline Location in R. Hippocampus & $t$-Stat & Location in R. Hippocampus & $t$-Stat \\
\hline Head, Lateral & 9.99 & Tail, Lateral & 8.04 \\
Tail, Medial & 7.55 & Body, Lateral & 6.45
\end{tabular}

\section{Discussion and Conclusions}

The question of whether better normalization of anatomy can improve the statistical power of fMRI analysis is not easily answered. Normalization error, it can be argued, is only one source of error that may fade in comparison to other sources, such as susceptibility artifacts. The fact that in an experiment where hippocampal activation is expected we detected more significant hippocampal activation with explicit normalization of the hippocampus than with whole-brain normalization, suggests that normalization error indeed had a significant impact on RFX statistics, and that methods which minimize it may increase the sensitivity and specificity of functional neuroimaging studies.

We do not presume that the $\mathrm{cm}$-rep method is the only approach capable of reducing normalization error in fMRI group studies. Indeed, non-parametric registration techniques, especially when driven by expert-placed landmarks (e.g., [5]), tend to register subcortical structures more accurately than parametric registration driven only by image forces. However, the $\mathrm{cm}$-rep method offers certain advantages for fMRI analysis that registration techniques do not. It makes it straightforward to associate voxel-wise features, such as functional activation, with shape features, such as the distance to the boundary. Thus, for instance, gray matter thickness can easily be used as a covariate in functional activation analysis, since functional voxels located in regions where grey matter is thicker are less likely to suffer from partial volume effects. The shape-based $\mathrm{cm}$ rep coordinate system also can be used to define anisotropic smoothing kernels that are thinner in the direction across the structure of interest, and longer in 
the direction along the structure. Such kernels can lead to structurally cohesive smoothing, which would reduce the amount of mixing of fMRI signal originating from different structures, and may strengthen the signal at locations adjacent to structures that do not activate. Finally, the $\mathrm{cm}$-rep deformable modeling approach, which allows incorporation of shape and intensity priors, may lead to an automatic segmentation algorithm, which would eliminate the need for binary masks. Our future work will focus on leveraging these potential advantages.

\section{Acknowledgement}

This work was supported by the NIH/NINDS R01 NS045839. We thank Prof. Charles L. Epstein, Prof. Jean Gallier and Marcelo Siqueira (Penn) for insightful discussions. We are indebted to Profs. Guido Gerig, Stephen M. Pizer, Sarang Joshi, and Martin Styner (UNC) for providing data and inspiration for this work.

\section{References}

1. J. Ashburner and K. Friston. Nonlinear spatial normalization using basis functions. Human Brain Mapping, 7(4):254-266, 1999.

2. A. Evans, D. Collins, S. Mills, E. Brown, R. Kelly, and T. Peters. 3d statistical neuroanatomical models from 305 mri volumes. In IEEE Nuclear Science Symposium and Medical Imaging Conference, pages 1813-1817, 1993.

3. B. Fischl, M. I. Sereno, and A. M. Dale. Cortical surface-based analysis II: inflation, flattening, and a surface-based coordinate system. NeuroImage, 9(2):195-207, 1999.

4. R. Frackowiak, K. Friston, C. Frith, R. Dolan, and J. Mazziotta, editors. Human brain function. Academic Press USA, 1997.

5. J. Haller, A. Banerjee, G. Christensen, M. Gado, S. Joshi, M. Miller, Y. Sheline, M. Vannier, and J. Csernansky. Three-dimensional hippocampal MR morphometry by high-dimensional transformation of a neuroanatomic atlas. Radiology, 202:504510, 1997.

6. A. Holmes and K. Friston. Generalisability, random effects and population inference. NeuroImage, 7:754, 1998.

7. S. M. Pizer, P. T. Fletcher, S. Joshi, A. Thall, J. Z. Chen, Y. Fridman, D. S. Fritsch, A. G. Gash, J. M. Glotzer, M. R. Jiroutek, C. Lu, K. E. Muller, G. Tracton, P. Yushkevich, and E. L. Chaney. Deformable m-reps for 3D medical image segmentation. International Journal of Computer Vision, 55(2):85-106, Nov 2003.

8. D. Tosun and J. L. Prince. Cortical surface alignment using geometry driven multispectral optical flow. In International Conference on Information Processing in Medical Imaging, pages 480-492, 2005.

9. P. Yushkevich, H. Zhang, and J. Gee. Parametric medial shape representation in 3 -D via the Poisson partial differential equation with non-linear boundary conditions. In G. Christensen and M. Sonka, editors, Information Processing in Medical Imaging, pages 162-173, 2005.

10. P. A. Yushkevich, H. Zhang, and J. C. Gee. Statistical modeling of shape and appearance using the continuous medial representation. In Medical Image Computing and Computer-Assisted Intervention, MICCAI, volume 2, pages 725-732, 2005.

11. M. M. Zeineh, S. A. Engel, P. M. Thompson, and S. Y. Bookheimer. Dynamics of the hippocampus during encoding and retrieval of face-name pairs. Science, 299(5606):577-80, Jan 2003. 